\title{
DESIGN STUDY OF A NEW BEAM SEPARATOR FOR PEEM3*
}

\author{
W. Wan, J. Feng and H. A. Padmore, LBNL Berkeley, CA 94720, USA
}

\section{Abstract}

Aberration correction of photoemission electron microscopes (PEEM) requires use of a magnetic beam separator. This device is a complex integrated magnetic system and is essentially fixed in its optical properties hence very susceptible to problems caused by mechanical or magnetic imperfections. Here we present a separate function design that is simple to construct, is fully adjustable and gives outstanding performance.

\section{INTRODUCTION}

Photo Emission Microscopy has become a powerful tool in recent years for the investigation of the surface chemical and magnetic properties of materials at high spatial resolution. While a spatial resolution of $50 \mathrm{~nm}$ can be achieved, this is at a huge penalty in terms of transmission. In addition, many scientific applications require a resolution significantly better than $50 \mathrm{~nm}$ and this has led to a need for aberration corrected microscope optics. Aberration correction is performed by an electron mirror that is tuned to have the same magnitude but opposite sign of the low order aberrations generated by the acceleration field and objective lens of the microscope. While being a successful strategy, it entails separation of the beams going into and coming out of the electron mirror and this has to date been accomplished by a $90^{\circ}$ magnetic separator. This device has itself to be aberration free to high order and therefore imposes very severe electron optical and engineering challenges. The design adopted in the SMART [1] and PEEM3 [2] microscopes use a single magnetic unit to achieve this separation (see Fig. 1 for the layout of PEEM3). It consists of 8 dipole magnets in each pass in a double symmetric design including 4 reverse bending magnets and employs only edge focusing. The performance of this unit depends critically on achieving very stringent machining tolerances and is essentially a untunable design.

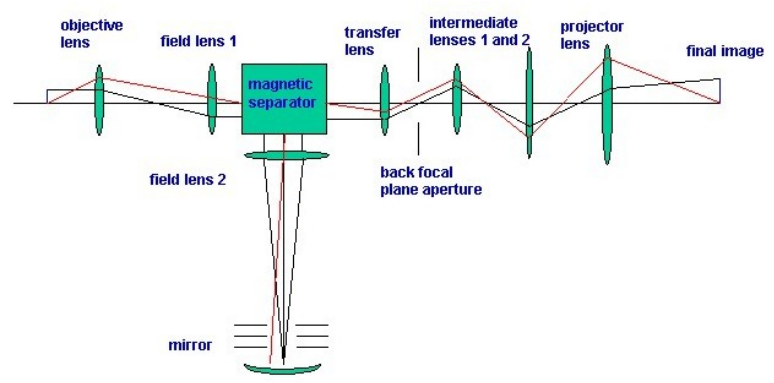

Figure 1: The layout of PEEM3.

In this work, we have examined a radically different ap-

* Work supported by U.S. Department of Energy, under Contract No. DE-AC03-76SF00098 proach. The magnetic dipole is simple and only has the function of guiding the beam. Focusing is achieved with a combination of electrostatic round lenses and quadrupoles. This device is thus fully tunable and much simpler to construct than a combined function separator magnet. Preliminary results on the layout, the Gaussian optics and the effect of the remaining aberrations the new separator are reported.

\section{LAYOUT AND GAUSSIAN OPTICS}

Instead of attempting to find a new design that cancels the same number of aberrations as the existing one, we tried to find a simpler design that fulfills the requirement for an aberration corrected PEEM with the resolution of a few nanometer such as SMART and PEEM3. We began with the simplest layout possible, which consists of just one bending magnet, as, for example, used in LEEMs [6]. Yet the large remaining dispersion after the two passes limits the resolution to hundreds of nanometer, which implies that the block containing the separator and the mirror column has to be achromatic. In the existing separator design, each pass of the separator is achromatic, which requires at least two bending magnets for each pass, or three in total. Due to the fact that the mirror column is a $-I$ transport, the entire separator-mirror block section is achromatic if $\eta^{\prime}=0$ after each pass. Concequently, only one bending magnet is needed. It should be pointed out that, in order to maintain mirror symmetry, the shared lenses (excluding the magnet) have to be electrostatic.

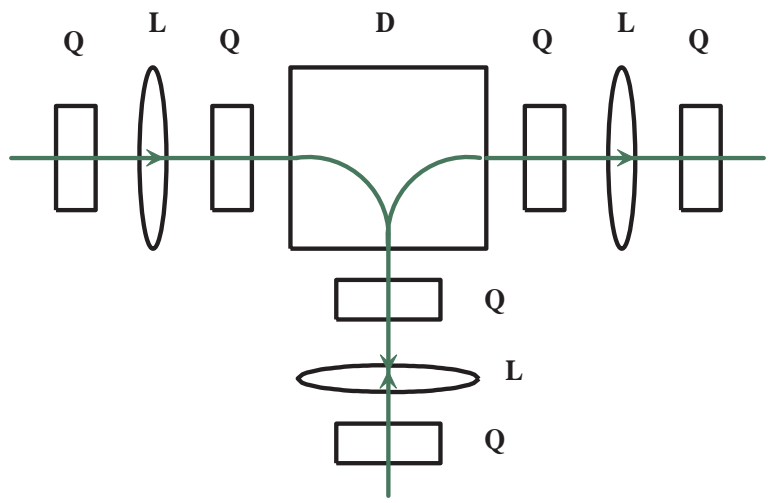

Figure 2: The layout of the new separator: $Q=$ quadrupole, $\mathrm{L}=$ round lens, $\mathrm{D}=$ dipole magnet.

Based on these findings, regular round lens, simple bending magnet and weak electrostatic quadrupole are chosen to form the new separator. The round lenses are chosen to be the main focusing lenses due to the fact that deviation from rotational symmetry caused by the bending magnet and the remaining aberrations of the lenses are small, resulting in weak quadrupoles. In order to cancel potentially dangerous aberrations such as coma, each pass is mirror symmetric 
about the center and forms a $-I$ transport and guarrentees that $\eta^{\prime}=0$ [7]. In summary, the field of the bending magnet alone defines the optical axis and four knobs are needed to ensure that $(x \mid \alpha),(y \mid \beta),(\alpha \mid x)$ and $(\beta \mid y)$ vanish, where $x, y$ are horizontal and vertical displacements, $\alpha$ and $\beta$ are horizontal and vertical slopes. As a result, two families of electrostatic quadrupoles are included in the new separator design and the position of the images along the optical axis is adjusted together with the strength of the round lenses. In total, the new separator consists of one bending magnet, three Einzal lenses and six electrostatic quadrupoles, as shown in Figure 2. The paraxial rays are shown in Figure 3 . It is worth noting that, compared to the original existing design, the new separator is fully tunable in terms of the total bending angle and the Gaussian optics. First, the total bending angle is determined by the magnetic field only and is independent of the Gaussian optics. Secondly, all four knobs needed for setting up the Gaussian optics can be adjusted individually. From the engineering point of view, the new separator requires a much simpler magnet and merely one third of the field for the existing separator, due to the absence of the reverse bending present in that design.

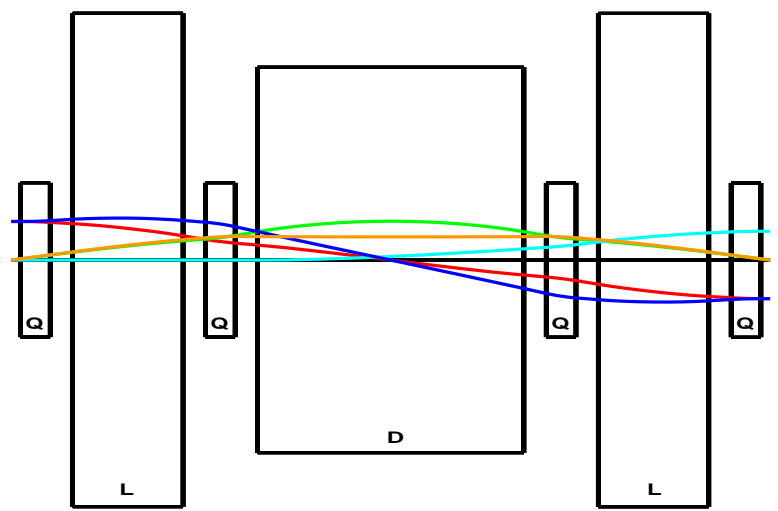

Figure 3: The fundamental rays of the $x-z$ (red, green and light blue) and $y-z$ (orange and dark blue) sections of an $90^{\circ}$ separator; displacement: $0.5 \mathrm{~mm}$, opening angle: 5 mrad, energy deviation: $0.5 \%$.

\section{FIELD MODELS AND ABERRATIONS}

In order to determine the performance of the new separator, aberrations have to be calculated and ray tracing has to be performed. Following our previous works on PEEM3 $[4,5,8]$, a model of the new separator has been developed using the differential algebraic (DA) [9] technique, which computes the Taylor map up to any order. The transfer map is in turn used for ray tracing; this is orders of magnitude faster than traditional methods. The field of the round lens, the magnet and the electrostatic quadrupoles are modeled using the charge ring method $[5,10]$, the SchwartzChristoffel model $[11,12]$ and the hypertagent function, respectively, which are implemented using the code COSY INFINITY [13].

With the field models implemented, it is straightforward to compute the aberrations. Listed in Table 1 are the key
Table 1: Selected aberrations (in millimeters) of the new and the existing separators together with the objective lens. Here $\alpha, \beta$ and $\kappa$ denote $p_{x} / p_{0}, p_{y} / p_{0}$ and $\delta E_{k} / E_{k 0}$, respectively. The distance between the first and the last image of the new separator is $28 \mathrm{~cm}$, which is the same as the existing one, yet the size of the bending magnet is only $13 \times 13 \mathrm{~cm}$

\begin{tabular}{|l|c|c|c|}
\hline & new & existing & objective lens \\
\hline$(x \mid \kappa)$ & 8 & 0 & 0 \\
\hline$(x \mid \alpha \kappa)$ & 146 & -82 & $-4.3 \times 10^{4}$ \\
\hline$(y \mid \beta \kappa)$ & 169 & -309 & $-4.3 \times 10^{4}$ \\
\hline$\left(x \mid \alpha^{2}\right)$ & -163 & 0 & 0 \\
\hline$\left(x \mid \beta^{2}\right)$ & -77 & 0 & 0 \\
\hline$(y \mid \alpha \beta)$ & -170 & 0 & 0 \\
\hline$\left(x \mid \alpha^{3}\right)$ & -1223 & 68 & $1.2 \times 10^{7}$ \\
\hline$\left(x \mid \alpha \beta^{2}\right)$ & -445 & -380 & $1.2 \times 10^{7}$ \\
\hline$\left(y \mid \alpha^{2} \beta\right)$ & -445 & -387 & $1.2 \times 10^{7}$ \\
\hline$\left(y \mid \beta^{3}\right)$ & -2035 & 7145 & $1.2 \times 10^{7}$ \\
\hline
\end{tabular}

aberrations of the new separator and the existing separator for single pass, as well as, for comparison, the aberrations of the objective lens are given. A few points are worth noting regarding this table. First, unlike the existing separator and all round lenses, the new design does have finite linear dispersion and second order geometrical aberration because it has only one symmetry plane. Since the mirror column is a $-I$ transport, both the linear dispersion and the second order axial aberration are cancelled after the second pass of the separator. Therefore, to first order, they do not affect the resolution. It does introduce more aberrations through coupling to other parts of the microscope. Yet, due to the weaker magnetic field, those extra terms are too small to significantly affect the point-to-point resolution (see Figure 4). Second, the chromatic and spherical aberrations of the existing and the new separators are of the same of order of magnitude, which are 2 and 4 orders of magnitude smaller than those of the objective lens, respectively. Third, the differences of the aberrations between the $x$ and $y$ planes are much smaller for the new separator than those for the existing separator, which indicates that the rotational symmetry is broken to a lesser degree in the new separator than in the existing separator. It is our believe that this also resulted from the weaker magnetic field.

\section{RESOLUTION WITHOUT ERRORS}

The overall performance of the new separator is determined by tracking an ensemble of secondary electrons through the microscope. The distribution of the electrons on the the sample is the same as the one used in the previous studies of PEEM3 [2, 4, 5, 8], which is based on the work of B. L. Henke, et al. [14]. The electrons are traced through the objective lens using the traditional numerical integration method, since the Taylor map diverges for an energy distribution of the secondary electrons that peaks at $1.3 \mathrm{eV}$ and extends from 0 to $20 \mathrm{eV}$. After the objective 
lens, the electrons are tracked through the 5th order Taylor map of the field lens computed using the DA technique and form an image after it. The result was bench marked against that from the finite difference and ray tracing code SIMION [15] and good agreement was found between the two models. Next, the 5th order Taylor map from the entrance to the exit of the separator, with the mirror column in between, and that from the entrance of the separator to the back focal plane after the separator are generated. An aperture is placed at the back focal plane to adjust the transmission. The distribution of the electrons that pass the aperture is analyzed at the exit of the separator to determine the resolution of the microscope, which is defined as the radius of the circle that encloses $68 \%$ of the total transmitted electrons $[2,4,5,8]$. The results for the new separator without errors are shown in Figures 4 and 5. As discussed above, the new and the existing separator have almost the same point-to-point resolution over the allowed range of transmission. Yet the new separator behaves much more isotropically when the source point is $15 \mu \mathrm{m}$ off the optical axis. It is our belief that this is the result of utilizing the the round lenses as the main focusing elements on the one hand and removing the reverse bending on the other.

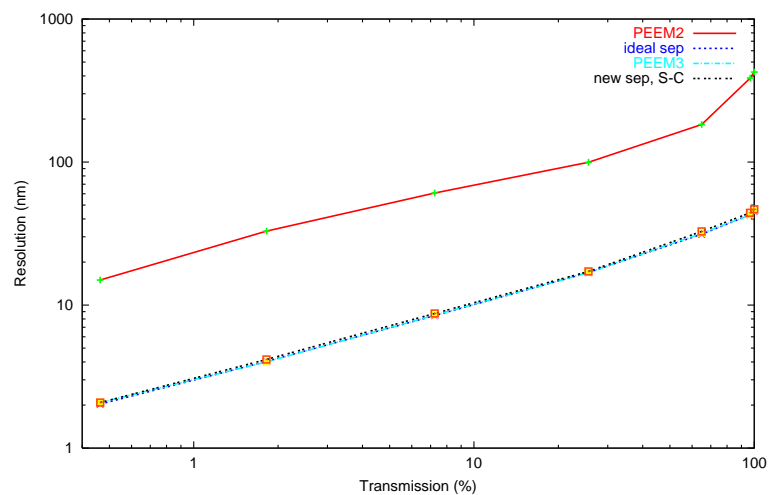

Figure 4: The resolutions of PEEM2, the ideal separator, PEEM3 separator and the new separator. The source is on the optical axis. Note that the ideal separator refers to the one with no aberrations. The same is true for all subsequent figures.

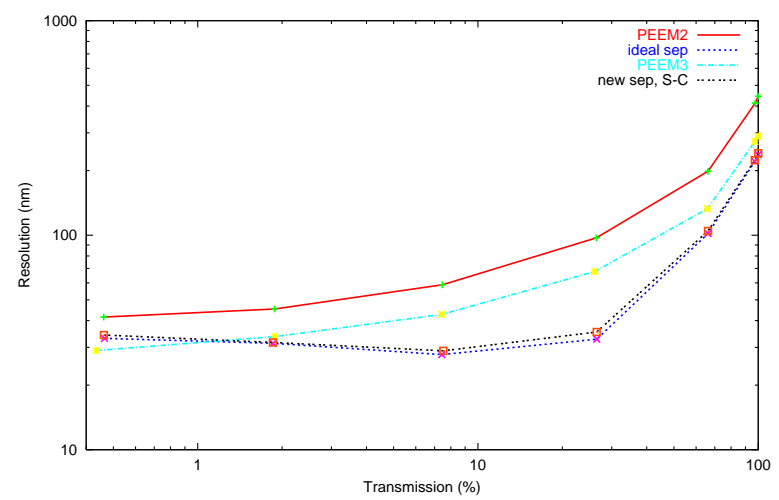

Figure 5: The resolutions of PEEM2, the ideal separator, PEEM3 separator and the new separator. The source is 15 $\mu \mathrm{m}$ off the optical axis vertically.

\section{FURTHER DISCUSSION}

In conclusion, a new design of separator is presented for aberration corrected photoemission microscopes. Compared to the existing design, the new separator has the following advantages. First, replacing edge focusing with separate lenses reduces the sensitivity of the Gaussian optics to the details of the magnetic field and increases the tunability of the separator. Second, using round lenses as the main focusing lenses, together with the reduction of the magnetic field due to the removal of reverse bending, helps to better preserve the rotational symmetry of the separator. One result is higher resolution and larger transmission for the source points that are off the axis. Another is relaxed tolerances on the stability of the power supplies and alignment which is not discussed here due to the limited space. A more detailed article will be published in the near future. It is worth noting that the tolarence on power supply stability and misalignment is roughly an order of magnitude more relaxed. On the whole, the new design simplifies the engineering design, lowers the total cost and increases tunability during operation.

\section{ACKNOWLEDGEMENTS}

The authors would like to thank J.-Y. Jung, A. MacDowell, M. A. Marcus, D. S. Robin, H. Rose, R. Schlueter, P. Schmid and A. Scholl for numerous useful discussions.

\section{REFERENCES}

[1] R. Fink et al., J. Electron Spectrosc. Relat. Phenom. 84, (1997) 231.

[2] J. Feng et al., J. Phys. Condens. Matter 17 (2005) S1339.

[3] H. Müller, D. Preikszas and H. Rose, J. Electron Microsc. 48 (1999) 191.

[4] Y. K. Wu et al., Nucl. Instrum. Methods Phys. Res. A 519 (2004) 230.

[5] W. Wan, J. Feng, H. A. Padmore and D. Robin, Nucl. Instrum. Methods Phys. Res. A 519 (2004) 222.

[6] P. Adamac, E. Bauer and B. Lencová, Rev. Sci. Instrum. 69, (1998) 3583.

[7] W. Wan and M. Berz, Phys. Rev. E 54, (1996) 2870.

[8] P. Schmid, et al., Rev. Sci. Instrum. 76, (2005) 023302.

[9] M. Berz, Part. Accel., 24, (1989) 109.

[10] D. R. Cruise, J. Appl. Phys. 34 (1963) 3477.

[11] R. Herzog, Z. Phys. 97 (1935) 596.

[12] H. Rose and D. Krahl, in Energy-Filtering Transmission Electron Microscopy, edited by L. Reimer (Springer, 1995) pp. 43.

[13] M. Berz, COSY INFINITY Version 8.1 Users Guide and Reference Manual, MSUHEP-20704, Dept. Physics and Astronomy, Michigan State University, 2002.

[14] B. L. Henke, J. A. Smith and D. T. Attwood, J. Appl. Phys. 48 (1977) 1852

[15] D. A. Dahl, J. E. Delmore and A. D. Appelhans, Rev. Sci. Instrum. 61 (1990) 601. 